\section{PEDAGOGÍA CRÍTICA PARA LA \\ CONCIENCIACIÓN EN TORNO A LA \\ DISCRIMINACIÓN DE GÉNERO Y LA \\ EMANCIPACIÓN DE LAS CHICAS EN EDUCACIÓN FÍSICA}

\author{
PEDAGOGÍA CRÍTICA PARA A CONSCIENTIZAÇÃO EM TORNO A \\ DISCRIMINAÇÃO DE GÊNERO E A EMANCIPAÇÃO DAS MENINAS EM \\ EDUCAÇÃO FÍSICA
}

\section{CRITICAL PEDAGOGY TO RAISE AWARENESS ABOUT GENDER \\ DISCRIMINATION AND GIRLS'EMANCIPATION IN PHYSICAL EDUCATION}

\section{Nuria Sánchez Hernández*, Susanna Soler Prat**, Daniel Martos García*}

Palabras clave: Educación Física. Construcción social del Género. Enseñanza. Feminismo.

Palavras chave: Educação Física. Construção social do Gênero.

Ensino.

Feminismo.

Keywords: Physical Education. Social construction of gender. Teaching. Feminism.

\begin{abstract}
Resumen: Este estudio pone encima de la mesa las posibilidades y limitaciones de la pedagogía crítica como herramienta de concienciación del alumnado de Educación Física en torno a las desigualdades de género. La innovación educativa constaba de 8 sesiones en las que se trabajaba el fútbol a través del aprendizaje cooperativo y los pretextos críticos encaminados a la reflexión. La investigación tomó un cariz etnográfico. Los resultados constataron cambios muy significativos en los chicos y sobre todo en las chicas, en aspectos actitudinales y relacionales. La mayoría de ellas pasaron del conformismo inicial a explicitar sus quejas y tomar las riendas de los grupos cooperativos. Esto nos hace albergar esperanzas sobre el potencial emancipador de la propuesta.
\end{abstract}

Resumo: No estudo que apresentamos, as possibilidades da pedagogia crítica como uma ferramenta para aumentar a conscientização dos estudantes de Educação Física sobre as desigualdades de gênero são postas na mesa. A inovação educacional consistiu em oito sessões de futebol por meio de aprendizado cooperativo e pretextos críticos voltados à reflexão. A pesquisa leva um olhar etnográfico. Os resultados mostraram mudanças muito significativas nas meninas em aspectos atitudinais e relacionais. Passaram do conformismo inicial para explicitar suas queixas e tomar as rédeas dos grupos cooperativos. Isso nos dá esperança para o potencial emancipador da proposta.

Abstract: This study intends to shed light on critical pedagogy's potential as a tool for raising Physical Education students' awareness about gender inequalities. Eight football lessons were taught using cooperative learning and critical pretexts to encourage reflection. The research used ethnographic techniques. The findings show some changes on girls' attitudinal and relational behaviour. Most of them gave up their initial conformism and started voicing their complaints and taking control of cooperative groups. These facts made us hopeful regarding the emancipatory possibilities of the proposition.
*Universidad de Valencia. Valencia, España.

E-mail:

nuria_esh@hotmail.com;

daniel.martos@uv.es

**Instituto Nacional de Educación Física de Cataluña. Barcelona,

España.

E-mail:

ssoler@gencat.cat

Recebido em: 28-10-2019 Aprovado em: 30-04-2020 Publicado em: 13-05-2020 


\section{INTRODUCCIÓN}

En pleno siglo $X X I$, el género continúa siendo una cuestión relevante en la Educación Física (EF) (SCRATON, 2018; SERRA et al., 2019; VALDIVIA-MORAL et al., 2012). Sin duda, es la asignatura donde se hacen más visibles tanto la reproducción de los modelos de masculinidad y feminidad hegemónicas, las relaciones de desigualdad y discriminación entre chicas y chicos, como la perpetuación de un modelo de actividad física y deporte claramente androcéntrico y patriarcal en el que todo aquello considerado tradicionalmente como masculino tiene más valor que aquello femenino (SUBIRATS; TOMÉ, 2007; VÁZQUEZ; ÁLVAREZ, 1990). En este sentido, diversos estudios constatan las relaciones de poder que se dan en EF y cómo estas afectan negativamente a las experiencias de muchas chicas en la práctica de actividad física y deporte (FLINTOFF; SCRATON, 2001; 2006; McCAUGHTRY, 2004). Así mismo, tal y como exponen Bramham (2003) o Lomas (2004), también afectan negativamente a las experiencias de aquellos chicos que no encajan en el modelo de masculinidad hegemónica.

En los contenidos del currículum que tienen mayor carga cultural de género, como el fútbol, es donde se pueden observar los comportamientos más estereotipados y discriminatorios (CLARK; PAECHTER, 2007; SKELTON, 2000; SOLER, 2009). Es precisamente este contexto el que convierte la EF en un campo de acción privilegiado para la transformación y el (posible) cambio de las relaciones tradicionales de género en la propia asignatura y a través de ella (SOLER, 2009).

Para ello, se hace necesario cuestionar esos modelos hegemónicos que, aunque no fijos, persisten a través de las creencias y experiencias del alumnado (CONNELL, 2006). Diversos estudios apuntan a la necesidad de desafiar las prácticas curriculares rígidas propias de nuestra área y cultura profesional (FLINTOFF; SCRATON, 2006; PENNEY, 2002). Se trata pues, de dejar de ver a las chicas como "el problema" y replantearse la propia estructura del currículum y la metodología que se pone en práctica en la EF (FLINTOFF, 2011; KIRK, 2010; KIRK; OLIVER, 2014; SÁNCHEZ-HERNÁNDEZ et al., 2018a; VERTINSKY, 1992; WRIGHT, 1997). En cierta manera, estos y otros estudios ponen encima de la mesa la necesidad de desarrollar una praxis feminista, es decir, una práctica en la cual ésta y la teoría vayan de la mano en pos de un objetivo común de transformación (MACDONALD, 2002).

Son varias las experiencias que desarrollan estrategias para desafiar las desigualdades de género presentes en la EF escolar (ENRIGHT; O'SULLIVAN, 2010; FITZPATRICK; RUSSELL, 2015; FUENTES-MIGUEL et al., 2018; GERDIN, PHILPOT; SMITH, 2018; GRAY; TREACY; HALL, 2017; KIRK et al., 2000; MITCHELL; GRAY; INCHLEY, 2015; OLIVER; HAMZEH, 2010). La mayoría de estas propuestas destacan la importancia de tener en cuenta la elección de las alumnas y darles voz y autonomía para lograr una transformación más duradera. Si los estereotipos de género siguen arraigados en las creencias de los y las alumnas se hace necesario analizarlos, cuestionarlos y debatirlos en clase (ENRIGHT; O'SULLIVAN, 2010). 
Desde este marco, se considera necesario tomar medidas para que el estudiantado reflexione, conozca las injusticias sociales y actúe en contra de las barreras que les impiden sentirse libres, tal y como se propone desde la pedagogía crítica.

La pedagogía crítica se plantea como una pedagogía preocupada por el empoderamiento personal y colectivo para provocar un cambio social, la lucha contra el autoritarismo, la búsqueda de la emancipación o la denuncia de las injusticias sociales (MUROS, 2006; SICILIA-CAMACHO; FERNÁNDEZ-BALBOA, 2009). Esta nueva perspectiva educativa está lejos de la tradicional, la que se preocupa sobre todo por adquirir conocimientos, competencias y habilidades (BOSCATTO; KUNZ, 2007; FERNÁNDEZ-BALBOA, 2004; PEDRAZ, 2013).

En este sentido, son fundamentales las aportaciones del brasileño Paulo Freire y sus trabajos dedicados, entre otras cosas, a la 'concientización'. Para él, la concientización era la única manera de evitar el dogmatismo y garantizar que, en su caso, 'los oprimidos' fueran actores principales en el proceso de desvelar la realidad y conocerla de forma crítica, incluso de recrear el propio conocimiento. Solo así, fruto de un proceso de concientización, las personas se comprometerían a luchar por su libertad (FREIRE, 2003). Sin embargo, existen pocos estudios que desarrollan la perspectiva freiriana en nuestra asignatura, como han señalado Nogueira et al. (2018). Entre estos, destacan las aportaciones de Ventorim (2000) y Silva y Francisco (2012).

A partir de estas consideraciones, y de acuerdo con las propuestas de MartosGarcía, Lorente-Catalán y Martínez Bonafé (2018), desde esta investigación se propone desarrollar y analizar una intervención pedagógica crítica, la cual pretende favorecer el empoderamiento personal de las chicas y transformar las relaciones de discriminación entre chicos y chicas, en las clases de EF y a través de la EF. Así, se trata de desarrollar en el alumnado la habilidad de interpretar críticamente la realidad que les rodea para, de esta forma, cuestionar los mitos y creencias que dan forma al conocimiento y a las experiencias que tenemos (MARTÍNEZ BONAFÉ, 1996). Con ello, volviendo al "para qué" que reclama la pedagogía, se pretende desvelar las injusticias sociales (concienciar) y contribuir de esta forma a la emancipación. Con esta intención, tal y como se describe detalladamente en Sánchez-Hernández et al. (2018b), se utilizan estrategias de aprendizaje cooperativo (y más concretamente la técnica de puzle de Aronson), y actividades de reflexión crítica, que denominamos pretextos críticos.

La técnica de puzle de Aronson, concretamente, se caracteriza por el trabajo en pequeños grupos y la existencia de una interdependencia positiva, donde cada persona aprende y cuida que el resto del grupo también aprenda (METZLER, 2005). Se pretende fomentar la solidaridad y respeto entre el alumnado (TRAVER; GARCÍA-LÓPEZ, 2006) así como la mentalidad grupal por encima de la individual, promoviendo el desarrollo de habilidades sociales (DYSON; LINEHAN; HASTIE, 2010; VELÁZQUEZ; FRAILE; LÓPEZ-PASTOR, 2014). En nuestro caso, el puzle de Aronson se usó en las últimas sesiones para el aprendizaje y desarrollo de las habilidades específicas del futbol, pero también para fomentar el respeto y la solidaridad entre el alumnado participante (SÁNCHEZ-HERNÁNDEZ et al., 2018b). 
Por otra parte, para aludir a este concepto de concientización, generar reflexión y desvelar las injusticias, también se utilizaron diversos pretextos críticos de tipo vivencial y textual, estrategia ya usada en diferentes estudios con orientaciones críticas (AZZARITO; SOLMON, 2006; MARTOS-GARCÍA et al., 2016; OLIVER, 2001). Estos pretextos se pueden alinear con las acciones comunicativas orientadas a la reflexión y el diálogo intersubjetivo (BOSCATTO; KUNZ, 2007).

Tal y como señalan Macdonald y Brooker (1999), la reflexión tiene una función provocativa que trata de romper con las injusticias sociales. Para ello, como apuntan Martínez Álvarez y García Monge (2002), es imprescindible que exista un clima de reflexión y diálogo adecuado, de escucha y de respeto, en que se pueda hablar con seguridad de los propios sentimientos y emociones. Sin este marco, las voces más oprimidas podrían quedar otra vez en silencio. En esta intervención los pretextos críticos que se plantearon y que se pueden encontrar descritos en SánchezHernández, Martos-Garcia y Soler (2018) y Sánchez-Hernández et al. (2018b), fueron: la formación de los equipos de una manera tradicional, una competición inicial sin pautas, la lectura de una historia ficticia sobre acoso escolar, y el visionado de vídeos sobre el futbol femenino. Con los textos y los documentos gráficos manteníamos la intención de, como sugiere Giroux (2001), rescatar aspectos sociales relevantes como la justicia social o el funcionamiento de las diferentes formas de poder.

En este artículo se presentan los resultados de esta intervención didáctica en relación a su capacidad de desvelar las injusticias sociales y cuestionar los modelos y las relaciones tradicionales de género por parte de las chicas que protagonizaron el estudio, así como las implicaciones en el desarrollo de las clases.

\section{METODOLOGÍA}

El estudio presentado forma parte de una etnografía crítica llevada a cabo en un instituto de Valencia (España) durante el curso escolar 2015-2016, durante la cual se desarrolló la Unidad Didáctica denominada "Estereotipos en fuera de juego". En esta experiencia participaron 5 grupos, involucrando a 111 chicos y chicas (de 14 y 15 años) y dos profesoras. El centro, como el barrio donde se encuentra, albergaba alumnado de clase media-baja y con una fuerte presencia en las aulas de personas migrantes. Las sesiones fueron diseñadas e impartidas por la primera investigadora.

La recolección de datos fue en línea con la perspectiva cualitativa y con los requisitos propios de la investigación educativa. Se llevó a cabo por distintas vías atendiendo a los requerimientos de triangulación de métodos e informantes. Todo el material que se recogió procedía de: (1) las respuestas individuales $(R)$ por parte de chicos y chicas a los ejercicios planteados antes, durante y después de la intervención; (2) los grupos de discusión entre el alumnado: al inicio de la UD (GDInicial) (sobre su propia experiencia en la práctica del fútbol, en la competición inicial, y la historia ficticia que se diseñó), y al final de la UD (GD-Final); y por último, (3) los diarios de campo donde se recogieron las observaciones durante las ocho sesiones de la intervención, tanto de la investigadora principal (DC-Núria), el amigo crítico (DC-Dani) y las profesoras (DC-Cris y DC-Maite). 
Todo el contenido fue transcrito y el análisis de los datos atendió a las fases que establecen Sparkes y Smith (2014) de codificación, categorización, establecimiento de subcategorías e interpretación de los datos, para la posterior creación de los informes finales. Todo el proceso de tratamiento de los datos se hizo de manera inductiva, manteniendo un orden coherente, como apunta Woods (1998). Así, tras identificar las ideas más importantes en relación a la temática estudiada, se creó una lista con 53 códigos que, después de una depuración, pasó a 14. En la parte final de descontextualización nos apoyamos en lo que describen Coffey y Atkinson (2005) acerca de extraer los datos y ubicarlos en las categorías pertinentes para ordenarlos y tratar toda la información.

En todos los casos, se contó con la aprobación de la dirección del centro, el departamento de EF y las familias del alumnado para el desarrollo de la experiencia y la recolección de datos. Además, se ha tratado de preservar la localización del centro y la identidad de las personas participantes, mediante el uso de códigos y pseudónimos. De acuerdo a los postulados de la investigación educativa, la experiencia trató en todo momento de no atentar contra la dignidad de las personas involucradas.

\section{RESULTADOS Y DISCUSIÓN}

Atendiendo a los resultados obtenidos hemos creído conveniente estructurarlos en dos bloques. El primero de ellos hace referencia al proceso de reflexión y diálogo crítico que da pie a la denuncia por parte de las chicas de la situación de injusticia y discriminación que experimentan, individualmente y como colectivo. El segundo, por un lado, se centra en cómo las chicas han ido tomado protagonismo en las clases a medida que la unidad didáctica iba avanzando y, por otro, en los cambios (o no), en sus actitudes y las relaciones entre ellas y los chicos de clase.

\subsection{LA DENUNCIA DE LA VIEJA DISCRIMINACIÓN: LAS CHICAS DESVELAN LA INJUSTICIA}

A través de los ejercicios de reflexión planteados al inicio de la UD, afloraron diversos sentimientos y actitudes que chicas y chicos tenían acerca del fútbol. En primera instancia, ellas hicieron referencia a la actitud de los chicos al no contar con ellas para practicar fútbol. Así lo refleja el comentario de Rosa: "[...] he conseguido quitar el balón a alguien y así he podido tocarlo. Venía hacia mí y lo he rozado. Una hora de partidos y 'casi tocar el balón' está bien” - apuntaba en tono irónico (GDInicial).

Este comentario refleja lo que apunta Soler (2009) acerca de que la mayoría de las chicas se sienten ignoradas por buena parte de los chicos en los juegos y actividades. Por ello, no es de extrañar que ellas mantengan esa desgana o poco interés en clase de fútbol motivada, entre otros aspectos, por el trato que reciben por los compañeros de clase (HILLS, 2006). Como bien explicaba Raquel: "[...] cuando te dicen los de tu propio equipo que te apartes porque les estorbas, ya ni te molestas por intervenir en clase" (GD-Inicial). Esta reflexión es compartida por la mayor parte de las chicas y refleja como el conflicto que se da en la pista, si no hay un espacio de 
reflexión, queda invisibilizado y la situación de exclusión pasa a normalizarse, tal y como apuntan también Oliver y Kirk (2015) o Tomé y Ruiz (1996).

Dada esta situación, ellas optan por desvincularse de las clases de EF porque no se les hace partícipes y adoptan una posición de poco compromiso, de pasotismo (ENRIGHT; O'SULLIVAN, 2010; SOLER, 2009). Un ejemplo que corrobora esta situación es el comentario de Fátima: "Prefiero que ni me miren, que me pase el balón por al lado y ni tocarlo, me da igual... Si lo tocas es peor porque empiezan a meterse contigo" (GD-Inicial).

Clark y Paechter (2007) explican cómo el grupo más hábil se burla y excluye tanto a las chicas como a los chicos que no saben jugar a fútbol. Esta situación esconde una relación de poder que consigue que las chicas, como se ha apuntado anteriormente, decidan no entrar en conflicto con los chicos y se mantengan al margen adoptando una postura de mirar y no participar (EVANS; DAVIES, 2004; SOLER, 2009; WRIGHT, 1997). Ellas adoptan una postura de indiferencia para no entrar en conflicto con los más hábiles, lo que directamente afecta a su falta de confianza a la hora de participar en clase (FLINTOFF; SCRATON, 2006). Se observa, pues, como las chicas en vez de hacer explícitas sus quejas por el hecho de ser excluidas del juego, adoptan formas de resistencia más pasivas como, por ejemplo, tratar de pasar desapercibidas, lo que se suele interpretar como falta de interés y motivación (SOLER, 2009; CHEPYATOR-THOMSON; ENNIS, 1997).

La creación de un clima de reflexión y diálogo entre chicos y chicas sobre qué sucedía en el juego, y cómo se sintieron jugando, permitió sacar a la luz las situaciones de discriminación y los efectos que tienen en el proceso de aprendizaje. Este hecho puede considerarse coherente con una pedagogía que busca la emancipación, por cuanto el uso del lenguaje verbal (en este caso por parte de las chicas para denunciar la discriminación ante la presencia de los chicos) favorece la producción de un pensamiento reflexivo y crítico, como apuntan Boscatto y Kunz (2007). Como decimos, algunas chicas denunciaron la desagradable situación que viven cuando juegan al fútbol, llegando incluso a ser insultadas por ellos, como por ejemplo Gemma: "[...] se piensan que no sabemos jugar, que somos malas... si fallamos nos recriminan... si no pasamos bien nos dicen de todo" $(R)$. El comentario de Martina ahonda en este hecho: "siempre nos menospreciáis, ya entramos a clase con esa desventaja. Así es muy difícil aprender" (GD-Final).

En estos espacios de debate se constató como las chicas son bien conscientes de los prejuicios de los cuales son víctimas y de cómo este hecho las limita y las condiciona a la hora de actuar. La denuncia del sexismo, e incluso de las relaciones de poder que se establecen, aflora públicamente mediante la voz de las propias chicas. El comentario de Natalia es bien elocuente: "[...] parece como si los chicos mandaran sobre nosotras... en qué posición ponernos, a quien pasar el balón..." (GD-Inicial). En otro grupo, Zaida, también lo denunció: "[...] claro que me gusta el fútbol, creo que soy de las pocas que trata de jugar, pero aun así parece como que tengo que pedirles permiso para jugar" (DC-Nuria).

Con estas declaraciones, en las que revelan las relaciones de dominio y subordinación que sufren en el juego, las chicas alzaron la voz para denunciar 
la discriminación y exclusión. Así, denotaron ser plenamente conscientes de ello y trataron de hacer partícipe al resto del grupo de esta situación. La creación de este marco de diálogo permitió a las chicas expresar la existencia del conflicto, evidenciando claramente los motivos que en muchos casos las llevan a actuar de forma pasiva. Con ello, se cuestiona la creencia de que no juegan porque no quieren, sino que no juegan porque no se da el contexto adecuado. Se trata, pues, de cambiar este contexto. $Y$ en este sentido es donde la metodología de aprendizaje cooperativo ofrece un nuevo marco en el que generar cambios en las relaciones entre chicos y chicas, tal y como se describe a continuación.

\subsection{UNA MAYOR EMANCIPACIÓN Y RESPETO: “ALGO DIFERENTE ESTABA PASANDO EN CLASE"}

Las actividades de reflexión previa, junto a la puesta en práctica de un modelo de aprendizaje cooperativo (en lugar de un modelo técnico y competitivo) implicó, como dijo una de las profesoras del grupo, que: "Algo diferente estaba pasando en clase" (DC-Cris).

El papel que adoptaron las chicas en clase de EF era totalmente nuevo para ellas. Así, manifestaron sentirse en cierto modo líderes de sus grupos y capaces de llevar el peso de la clase. Muestra de ello es el comentario de Patri: "[...] expliqué el ejercicio sola "ale va, vamos al tema, me toca a mí explicar el control y el pase, vosotros dos poner los conos y el resto a por los balones. Prestaron atención e hicieron lo que les mandé sin rechistar" $(R)$.

Mediante la técnica de puzle de Aronson propia del aprendizaje cooperativo las chicas pasaron a tomar la iniciativa cuando les correspondía: "Veo a algunas chicas coger la responsabilidad de sus grupos, papel en mano... incluso una alumna reactiva al fútbol está participando" (DC-Dani). Así mismo empezaron a sentirse seguras y realizadas por haber logrado los objetivos, como apunta Frida: "Yo me veía incapaz de explicar un ejercicio de fútbol, incapaz. Ahora siento satisfacción de saber qué soy capaz, me siento reconfortada, fuerte en fútbol. Me siento llena” (GD-Final).

Otra chica, Lola, también valora la experiencia de forma muy positiva: “ $[\ldots]$ ahora te sientes como necesaria. En los partidos, si te vas no pasa nada, pero aquí tienes que prestar atención para explicar a todo el grupo las sesiones porque mejor que tu no las sabe nadie" (GD-Final). Los comentarios ponen de manifiesto la creación de un sentimiento de grupo en el que ellas se sienten parte indispensable, al contrario de lo que suele ocurrir en las actividades bajo un modelo más técnico y tradicional. Tanto es así que sus propios compañeros también advirtieron el cambio, como explica Marcos: “[...]se sentían útiles al darles el papel de entrenadoras, puede que necesitaran esa motivación" (GD-Final).

Tal y como apuntan Kirk et al. (2000) o Davis (2003), entre otros estudios, para transformar las relaciones de género es importante hacer partícipes a las chicas en clase, darles protagonismo y responsabilidad, facilitándoles así la oportunidad de vivenciar el liderazgo y mejorar su autoestima y autoconfianza. En añadidura, desde una posición alineada con la pedagogía crítica, las actuaciones de índole 
transformadora deben combinar la reflexión y la práctica, es decir, donde se reflexione sobre la práctica y donde la práctica derive de una reflexión (NOGUEIRA, 2003), como es el caso.

Sin embargo, a pesar de los cambios en la metodología y el sistema de relaciones, puede haber diferentes grados de éxito, como también apunta Flintoff (2011). Un ejemplo de ello es la constatación de Mónica quien, después de la experiencia, expresaba: "Juro que lo he intentado, pedir el balón, no perderla... esta vez sí que tenía intención de cambiar, pero ellos siguen metiéndose conmigo cuando toco el balón, ya no sé qué más hacer" (GD-Final). En esta línea, Nicky señalaba: "empezó enseñándome a mí sola, pero al ver que no me salía el ejercicio, él cogió el balón y empezó a hablarme mal. Menudo horror" (GD-Final).

No obstante, los casos de exclusión y discriminación fueron menos frecuentes que en anteriores ocasiones. Las actividades de reflexión previas facilitaron el cambio de actitud entre los chicos, según la opinión de Paula: "Ahora si un chico te tiene que corregir encuentra las palabras para decírtelo bien. Al final saben que el camino no es insultar, ya se lo dijimos en las clases teóricas que nos hacían sentir fatal y han cambiado. Encuentran la forma de decírtelo" (GD-Final). Frida también destacó este hecho: "Al escucharnos contar lo mal que nos sentimos cuando nos tratan como a un pegote, ellos se han puesto en nuestro lugar y parece que han cambiado su mentalidad" (R). Nos parece importante este cambio, por lo que añadimos otro comentario de Fátima al respecto: "Parece que ellos han cambiado, están irreconocibles" (DCDani). Las observaciones de la propia profesora corroboraron las impresiones de las chicas: "se les ve disfrutar, reírse, comunicarse, miradas de complicidad entre ellos" (DC-Maite).

Gracias a las estrategias de reflexión desarrolladas con el alumnado, algunos chicos parecieron empatizar con el sentimiento de desagravio de las chicas, tal y como describe Oliver (2001). Así, la mayoría de los chicos, a lo largo de este proceso modificaron su actitud, pasando de las formas violentas que sus compañeras habían denunciado a formas más colaborativas.

Al igual que en el trabajo de Gray, Treacy y Hall (2017) la creación de un entorno de diálogo y confianza entre chicos y chicas permitió mejorar las actitudes y relaciones entre todo el grupo. La propuesta metodológica basada en el aprendizaje cooperativo y la reflexión crítica, si bien en algunos casos no logró cambiar las actitudes de insultos o reproches de algunos chicos, logró un cambio significativo en el clima de clase. Las propias chicas reconocieron este cambio, como se desprende de las palabras de Laura: "Hemos creado un buen ambiente en clase... antes no me gustaba el fútbol, pero ahora sí, aunque me llevé un balonazo" (GD-Final).

\section{COMENTARIOS FINALES}

El contenido del fútbol es habitual en las programaciones de EF aunque, debido precisamente a su fuerte carga cultural de género, en algunos casos se instrumentaliza su uso o, directamente, se elimina de las programaciones (SÁNCHEZ-HERNÁNDEZ; MARTOS-GARCÍA; SOLER, 2019). Si bien esta postura evita la puesta en escena en 
las clases de EF de las relaciones tradicionales de género, ello no permite abordar dicha problemática, de modo que, por omisión, se siguen perpetuando los modelos hegemónicos. Además, frustra que las alumnas se desenvuelvan con garantías en los deportes colectivos, entendidos normalmente como instrumentos de poder al servicio tradicionalmente de los alumnos (CHANN-VIANA; MOURA; MOURÃO, 2010).

En esta investigación, siguiendo las exigencias de concientización de Freire (2003), se expone como mediante una metodología transformadora basada en el aprendizaje cooperativo y la reflexión crítica, las clases de EF pueden convertirse en una oportunidad para desvelar las injusticias y la discriminación de género para aquellos chicos y chicas que no eran conscientes de ello. De acuerdo con Azzarito (2009) y Dowling y Garrett (2017), los procesos de concienciación que se pueden dar en EF son herramientas de resistencia que hacen posible la emancipación de los colectivos oprimidos. Además, se da buena prueba de cómo la EF puede combinar la práctica y la reflexión, como demanda Nogueira (2013), donde una dialoga con la otra.

Sin duda, una sola UD no es suficiente para transformar las relaciones de género arraigadas en el día a día del alumnado, pero la creación de espacios donde las chicas, como grupo oprimido en este contexto, alcen la voz, denuncien las situaciones de desigualdad que estaban sufriendo en la práctica del fútbol, y tomen protagonismo en las clases, es un paso hacia dicha emancipación.

Por otra parte, es necesario destacar también algunas de las limitaciones de la investigación. Entre otros aspectos, hubiera sido interesante observar el desarrollo de la competición de fútbol después de la intervención, tal y como se realizó en la primera sesión y que dio pie al primer pretexto crítico y al primer debate. Así se podría haber analizado si los cambios en el clima de la clase se dan solo en el marco de actividades cooperativas planificadas por el profesorado, o si estos cambios se trasladan también a la práctica de actividades de competición sin la tutela del profesor o profesora. Por otra parte, también se constató que hubo aspectos que no aparecieron en el debate como, por ejemplo, los insultos como "marimacho" o las etiquetas "jugar como una niña", y que otros estudios han constatado que están presentes entre las creencias del alumnado (CLARK; PAECHTLER, 2007; MONFORTE; ÚBEDA-COLOMER, 2019). Se observa, consecuentemente, la necesidad de incorporar pretextos críticos explícitos respecto a estos prejuicios para abordarlos y desmontarlos, entre otros aspectos.

Por otra parte, en los datos que recogimos y que no hemos tratado en profundidad en este artículo por cuestiones de espacio, se constató como la línea que delimita a los colectivos vulnerados no coincide siempre con la del género; dicho de otra forma, el género no explica todas las discriminaciones (CHANN-VIANA; MOURA; MOURÃO, 2010). Así, mientras muchas chicas hábiles tenían buena relación con la $E F$, aparecieron testimonios de chicos marginados, tanto en las sesiones en general como en las de futbol en particular. Aunque el género es una variable fundamental y la praxis feminista se nos revela necesaria, hemos de tratar de ampliar las miras y flexibilizar las líneas que definen las categorías de personas con las que tratamos. Tratándose de pedagogía crítica, la raza, la diversidad funcional o la habilidad motriz 
juegan un papel protagonista, por lo que atender la interseccionalidad de todas estas variables es muy pertinente para atacar las desigualdades (FLINTOFF; FITZGERALD; SCRATON, 2008).

Finalmente, esperamos que esta experiencia impulse el desarrollo de nuevas propuestas transformadoras como formas de praxis feministas. Dichas praxis son necesarias, entendemos, para educar en género al alumnado y trastocar así las relaciones tradicionales que chicos y chicas establecen en EF, y haciendo visible lo que se encontraba oculto.

\section{REFERENCIAS}

AZZARITO, Laura; SOLMON, Melinda A. A Feminist Poststructuralist View on Student Bodies in Physical Education: Sites of Compliance, Resistance, and Transformation. Journal of Teaching in Physical Education, v. 25, n. 2, p. 200-225, 2006.

AZZARITO, Laura. The Panopticon of physical education: pretty, active and ideally white. Physical Education and Sport Pedagogy, v. 14, n. 1, p. 19-39, 2009.

BOSCATTO, Juliano Daniel; KUNZ, Elenor. Contribuições teóricas para uma didática comunicativa na Educação Física escolar. Motrivivência, v. 19, n. 28, p.101-114, 2007.

CHAN-VIANNA, Alexandre Jackson; MOURA Diego Luz; MOURÃO Ludmila. Educación Física, género y escuela: un análisis de la producción académica. Movimento, v. 16, n. 2, p. 149-166, 2010.

CHEPYATOR-THOMSON, Jepkorir R.; ENNIS, Catherine D. Reproduction and Resistance to the Culture of Femininity and Masculinity in Secondary School Physical Education.

Research Quarterly for Exercise and Sport, v. 68, n. 1, p. 89-99, 1997.

CLARK, Sheryl; PAECHTER, Carrie. 'Why can't girls play football?' Gender dynamics and the playground. Sport, Education and Society, v. 12, n. 3, p. 261-276, 2007.

COFFEY, Amanda; ATKINSON, Paul. Encontrar el sentido a los datos cualitativos. Alicante: Universidad de Alicante, 2005.

CONNELL, Robert W. Understanding men: gender sociology and the new international research on masculinities. In: SKELTON, Christine; FRANCIS, Becky; SMULYAN, Lisa (eds.). Handbook of Gender and Education. London: Sage, 2006. p.18-30.

DAVIS, K Kathryn L. Teaching for Gender Equity in Physical Education: A Review of the Literature. Women in Sport and Physical Activity Journal, v. 12, n.2, p. 55-81, 2003.

DYSON, Ben P.; LINEHAN, Nicole R.; HASTIE, Peter A. The Ecology of Cooperative Learning in Elementary Physical Education Classes. Journal of Teaching in Physical Education, v. 29, n. 2, p. 113-130, 2010.

DOWLING, Fiona; GARRETT, Robyne. The Transformative possibilities of Narrative Inquiry. In: ENNIS, Catherine (ed.). Handbook of Physical Education Pedagogies. London: Routledge, 2017. p. 332-342.

ENRIGHT, Eimear; O'SULLIVAN, Mary. 'Can I do it in my pyjamas?' Negotiating a physical education curriculum with teenage girls. European Physical Education Review, v. 16, n. 3 , p. 203-222, 2010. 
EVANS, John; DAVIES, Bronwyn. Pedagogy, symbolic control, identity and health. In: EVANS, John; DAVIES, Bronwyn; WRIGHT, John (eds.). Body Knowledge and Control: Studies in the Sociology of Physical Education and Health. London: Routledge, 2004. p. 3-18.

FERNÁNDEZ-BALBOA, Juan Miguel. Recuperando el valor ético-político de la pedagogía: Las diferencias entre la pedagogía y la didáctica. En: FRAILE, Antonio (coord.). Didáctica de la Educación Física. Una perspectiva crítica y transversal. Madrid: Biblioteca Nueva, 2004. p. 315-330.

FITZPATRICK, Katie; RUSSELL, Dan. On being critical in health and physical education, Physical Education and Sport Pedagogy, v. 20, n. 2, p. 159-173, 2015.

FLINTOFF, Anne. Gender and learning in PE and youth sport. In: ARMOUR, Kathleen (ed.). Sports Pedagogy: An Introduction for Teaching and Coaching. London: Pearson, 2011. p. 161-179.

FLINTOFF, Anne; SCRATON, Sheila. Stepping into active leisure? Young women's perceptions of active lifestyles and their experiences of school physical education. Sport, Education and Society, v. 6, n. 1, p. 5-21, 2001.

FLINTOFF, Anne; SCRATON, Sheila. Girls and PE. In: KIRK, David; MACDONALD, Doune; O' SULLIVAN, Mary (eds.). The Handbook of Physical Education. London: Sage, 2006. p. 767-783.

FLINTOFF, Anne; FITZGERALD, Hayley; SCRATON, Sheila. The challenges of intersectionality: researching difference in physical education. International Studies in Sociology of Education, v. 18, n. 2, p. 73-85, 2008.

FUENTES-MIGUEL, Jorge et al. Más allá del binario sexo/género: una propuesta pedagógica trans-queer en Educación Física. In: LORENTE-CATALÁN, Eloísa; MARTOSGARCÍA, Daniel (eds.). Educación física y pedagogía crítica. Propuesta para la transformación personal y social. Lleida: Universitat de Lleida, 2018. p. 167-192.

FREIRE, Paulo. Pedagogía del oprimido. Madrid: Siglo XXI de España , 2003.

GERDIN, Goran; PHILPOT, Rod; SMITH, Wayne. It is only an intervention, but it can sow very fertile seeds: graduate physical education teachers' interpretations of critical pedagogy. Sport, Education and Society, v. 23, n. 3, p. 203-215, 2018.

GIROUX, Henry. Cultura, política y práctica educativa. Barcelona: Graó, 2001.

GRAY, Shirley; TREACY, Jennifer; HALL, Edward. Re-engaging disengaged pupils in physical education: an appreciative inquiry perspective. Sport, Education and Society, v. 23, n. 3, p. 1-15, 2017.

HILLS, Laura A. Playing the Field(s): An Exploration of Change, Conformity and Conflict in Girls' Understandings of Gendered Physicality in Physical Education. Gender and Education, v. 18, n. 5, p. 539-556, 2006.

KIRK, David. Why research matters: Current status and future trends in physical education pedagogy, Movimento, v. 16, n. 2, p. 11-43, 2010.

KIRK, David; OLIVER, Kimberly. The same old story: the reproduction and recycling of a dominant narrative in research on physical education for girls. Apunts: Educación Física y Deportes, v. 116 n. 2, p. 7-22, 2014.

KIRK, David et al. Towards Girl-Friendly Physical Education: the Nike/Youth Sport Trust Girls in Partnership Project, Final Report. Loughborough: Institute of Youth Sport. 2000. 
LOMAS, Carlos (ed.). Los chicos también Iloran: identidades masculinas, igualdad entre los sexos y coeducación. Barcelona: Paidós, 2004.

MACDONALD, Doune. Extending agendas: physical culture research for the twenty-first century. In: PENNEY, Don (ed.). Gender and Physical Education: Contemporary Issues and Future Directions. London: Routledge, 2002. p. 208-222.

MACDONALD Doune; BROOKER, Rose. Articulating a critical pedagogy in physical education teacher education. Journal of Sport Pedagogy, v. 5, n. 1, p. 51-63, 1999.

MARTÍNEZ ÁLVAREZ, Lucio; GARCÍA MONGE, Alfonso. Reflexionar sobre el género desde escenas de práctica escolar. Apunts. Educació Física i Esports, v. 3, n. 69, p. 118-123, 2002.

MARTíneZ BONAFÉ, Jaume. Poder y conciencia. Cuadernos de Pedagogía, v. 253, p. 78-84, 1996.

MARTOS-GARCÍA, Daniel; LORENTE-CATALÁN, Eloísa; MARTÍNEZ BONAFÉ, Jaume. Educación física y pedagogía crítica: una necesidad educativa. In: LORENTE-CATALÁN, Eloísa; MARTOS-GARCÍA, Daniel (eds.). Educación física y pedagogía crítica.

Propuesta para la transformación personal y social. Lleida: Universitat de Lleida, 2018. p. 29-46.

MARTOS-GARCIA, Daniel et al. 'Huesos de cristal' y Educación Física. Una experiencia de simulación e imaginación hacia la inclusión. Cultura, Ciencia y Deporte, v. 33, n. 11, p. 225-234, 2016.

McCAUGHTRY, Nate. Learning to read gender relations in schooling: implications of personal history and teaching context on identifying disempowerment for girls. Research Quarterly for Exercise and Sport, v. 75, n. 4, p. 400-412, 2004.

METZLER, Michael. Instructional models for physical education. Scottsdale, AZ: Holcomb Hathaway, 2005.

MITCHELL, Fiona; GRAY, Shirley; INCHLEY, Joe. 'This choice thing really works'. Changes in experiences and engagement of adolescent girls in physical education classes, during a school-based physical activity programme. Physical Education and Sport Pedagogy, v. 20, n. 6, p. 593-611, 2015.

MONFORTE, Javier; ÚBEDA-COLOMER, Joan. 'Como una chica: un estudio provocativo sobre estereotipos de género en educación física. Retos, n. 36, p. 74-79, 2019.

MUROS, Beatriz. La puesta en práctica de la Pedagogía Crítica: estrategias metodológicas críticas. Tàndem. Didáctica de la Educación Física, v. 20, p. 33-43, 2006.

NOGUEIRA, Quéfren Weld Cardozo. Educação física e pedagogia crítica: praticar o discurso? Perspectiva, v. 21, n. 1, p. 179-197, 2003.

NOGUEIRA, Valdilene Aline; MALDONADO, Daniel Teixeira; SILVA, Sheila Aparecida Santos; FREIRE, Elisabete dos Santos; MIRANDA, Maria Luiza de Jesus. Práticas corporais e Paulo Freire: Uma análise sobre a produção do Conhecimento. Movimento, v. 24, n. 4, p. 1265-1280, 2018.

OLIVER, Kimberly L. Images of the Body from Popular Culture: Engaging Adolescent Girls in Critical Inquiry. Sport, Education and Society, v. 6, n. 2, p. 143-164, 2001.

OLIVER, Kimberly L; HAMZEH, Manal. "The boys won't let us play": 5th grade mestizas challenge physical activity discourse at school'. Research Quarterly for Exercise and Sport, v. 81, n. 1, p. 39-55, 2010. 
OLIVER, Kimberly; KIRK, David. Girls, gender and Physical education. An activist approach. London: Routledge, 2015.

PEDRAZ, Miguel V. Crítica de la educación física y Educación Física Crítica en España. Estado (crítico) de la cuestión. Movimento, v. 19, n. 1, p. 309-329, 2013.

PENNEY, Dawn (ed.). Gender and physical education: contemporary issues and future directions. London: Routledge, 2002.

SÁNCHEZ-HERNÁNDEZ, Nuria; MARTOS-GARCÍA, Daniel; SOLER, Susanna. ¡Otra vez el maldito fútbol, lo odio! De la reproducción a la transformación. Tándem. Didáctica de la Educación Física, v. 60, p. 43-49, 2018.

SÁNCHEZ-HERNÁNDEZ, Nuria; MARTOS-GARCÍA, Daniel; SOLER, Susanna. La (re) construcción de la identidad profesional de las profesoras de EF: de la complicidad y la reproducción de las relaciones de género a la disposición al cambio educativo. Revista Complutense de Educación, v. 30, n. 3, p. 879-893, 2019.

SÁNCHEZ-HERNÁNDEZ, Nuria et al. Challenging gender relations in PE through cooperative learning and critical reflection. Sport, Education and Society, v. 23, n. 8, p. 812-823, 2018a.

SÁNCHEZ-HERNÁNDEZ, Nuria et al. Estereotipos de género, futbol y aprendizaje cooperativo: una propuesta crítica para Secundaria. In: LORENTE-CATALÁN, Eloísa; MARTOS-GARCIIA, Daniel (eds.). Educación física y pedagogía crítica. Propuesta para la transformación personal y social. Lleida: Universitat de Lleida, 2018b. p. 293-321.

SCRATON, Sheila. Feminism and PE: Does Gender Still Matter? In: MANSFIELD, Louise; CAUDWELL, Jayne; WHEATON, Belinda; WATSON, Beccy (eds.), The Palgrave Handbook of Feminism and Sport, Leisure and Physical Education. London: Palgrave Macmillan UK, 2018. p. 25-42.

SERRA, Pedrona et al. Masculinización en estudios de Ciencias de la Actividad Física y el Deporte. Apunts. Educació Física i Esports, v. 135, n. 1, p. 9-25, 2019.

SICILIA-CAMACHO, Álvaro; Fernández-Balboa, Juan Miguel. Reflecting on the moral bases of critical pedagogy in PETE: toward a Foucaultian perspective on ethics and the care of the self. Sport, Education and Society, v. 14, n. 4, p. 443-463, 2009.

SILVA, Sabrina Melo Correia; FRANCISCO, Marcos Vinícius. Educação Física Escolar e autonomia: reflexões sobre as contribuições da teoria histórico-cultural. Colloquium Humanarum, v. 9, n. 2, p. 66-72, 2012.

SKELTON, Christine. 'A Passion for Football': Dominant Masculinities and Primary Schooling, Sport, Education and Society, v. 5, n. 1, p. 5-18, 2000.

SOLER, Susanna. Los procesos de reproducción, resistencia y cambio de las relaciones tradicionales de género en la educación física: el caso del fútbol. Cultura y Educación, v. 21, n. 1, p. 31-42, 2009.

SPARKES, Andrew; SMITH, Brett. Qualitative Research Methods in Sport, Exercise and Health. London: Routledge, 2014.

SUBIRATS, Marina; TOMÉ, Amparo. Balones fuera. Reconstruir los espacios desde la coeducación. Barcelona: Octaedro Ediciones, 2007.

TOMÉ, Amparo; RUIZ; Rafael Antonio. El espacio de juego: escenario de relaciones de poder. Aula, el juego como instrumento educativo, v. 52-53, p. 37-41, 1996. 
TRAVER, Joan Andrés; GARCÍA-LÓPEZ, Rafaela. La técnica puzzle de Aronson como herramienta para desarrollar la competencia "compromiso ético" y la solidaridad en la enseñanza universitaria. Revista Iberoamericana de Educación, v. 40, n. 4, p. 1-9, 2006.

VÁZQUEZ, Benilde; ÁLVAREZ, Gonzalo (eds.). Guía para una educación física no sexista. Madrid: Ministerio de Asuntos Sociales-Instituto de la Mujer, 1990.

VALDIVIA-MORAL, Pedro Ángel et al. Concepto de coeducación en el profesorado de Educación Física y metodología utilizada para su trabajo. Movimento, v. 18, n. 4, p. 197217, 2012.

VELÁZQUEZ, Carlos; FRAILE, Antonio; LÓPEZ-PASTOR, Víctor Manuel. Aprendizaje cooperativo en Educación Física. Movimento, v. 20, n. 1, p. 239-259, 2014.

VENTORIM, Silvana. Caracterização do esporte segundo a orientação didático-pedagógica da teoria de Paulo Freire. Motrivivência, v. 11, n. 14, p. 187-198, 2000.

VERTINSKY, Patricia. Reclaiming space, revisioning the body: the quest for gendersensitive physical education. Quest, v. 44, n. 3, p. 373-396, 1992.

WOODS, Peter. La escuela por dentro: la etnografía en la investigación educativa. Madrid: Paidós/MEC, 1998.

WRIGHT, Jan. The Construction of Gendered Contexts in Single Sex and co- Educational Physical Education Lessons. Sport, Education and Society, v. 2, n. 1, p. 55-72, 1997. 